\title{
La enseñanza-aprendizaje de la investigación cualitativa en el medio sanitario
}

\author{
Teaching and learning the science of qualitative research \\ in the health area
}

Abstract Qualitative research constitutes a necessary perspective of knowledge within the field of health services. Healthcare always occurs in complex contexts and its enhancement requires research methodologies that address this complexity. Nevertheless, the knowledge and use of qualitative research in health services is still very limited. Among the different factors that affect its development, the teaching and learning of qualitative research proves to be fundamental, even beyond undergraduate education. Healthcare professionals and health services present certain specific aspects that must be considered in the design and development of the teaching and learning of qualitative research. Based on an eightyear online training experience with Primary Healthcare professionals, the main challenges are indentified and discussed.

Key words Qualitative research, Teaching, Learning, Health services
Resumen La IC constituye una perspectiva de conocimiento necesaria en el medio sanitario. El trabajo asistencial se lleva a cabo siempre en contextos complejos y su mejora requiere de metodologías de investigación que asuman dicha complejidad. Sin embargo, el conocimiento y la puesta en práctica de la investigación cualitativa (IC) en los servicios de salud continúan siendo muy limitados. Entre los diversos factores que condicionan su desarrollo, la enseñanza-aprendizaje (EA) de la IC resulta fundamental, y no sólo a nivel de pre-grado. Los profesionales y el medio sanitario presentan determinadas particularidades que han de ser tenidas en cuenta en el diseño y desarrollo de la E-A orientada a dicho campo. A partir de una experiencia formativa on-line durante ocho años para profesionales de Atención Primaria, se discuten los principales retos identificados al respecto.

Palavras-chave Investigación cualitativa, Enseñanza, Aprendizaje, Servicios de salud 
Enseñanza-aprendizaje de la IC: para qué, para quién y cómo

La importancia y las dificultades vinculadas a la enseñanza-aprendizaje (E-A) de la IC han sido objeto de publicaciones, simposios y reuniones de trabajo a nivel internacional, y también en el área iberoamericana ${ }^{1-3}$. Recopilaciones de la bibliografía nos muestran a su vez la gran heterogeneidad de las disciplinas y de las áreas temáticas involucradas ${ }^{4,5}$. La E-A de la IC reproduce en gran medida la propia pluralidad de la IC en cuanto área de encuentro de diferentes ámbitos profesionales, corrientes de pensamiento, y enfoques del quehacer investigador. En ocasiones los enfoques se orientan más hacia los niveles teóricos y epistemológicos, en muchos de los casos se centran en los métodos y procedimientos de generación de información y de análisis, y en general los orígenes de las aportaciones se sitúan en la sociología, en la psicología, en la educación, $y$ en menor medida en las ciencias de la salud. Ante las tendencias a reducir y ordenar la E-A de la IC en función de sus procedimientos, Hammersley agrupaba las respuestas en tres modelos alternativos de E-A que denominaba como artesanía, profesión, y bricolaje ${ }^{6}$, ejemplos a su vez de la heterogeneidad que caracteriza a la IC.

Más adelante volveremos a referirnos a dicha diversidad de enfoques y corrientes como uno de los retos a tener en cuenta en el diseño de los programas formativos. Pero la correspondencia entre E-A e IC no se limita a la pluralidad. Si el desarrollo de la IC requiere de una pregunta y objetivos relevantes para las poblaciones a quienes va dirigida, y del diseño flexible del proceso a seguir en función de aquéllos, también en la E-A los programas y dinámicas han de tener en cuenta las finalidades, expectativas y características de los discentes y docentes implicados en cada caso. De ahí la importancia, en mi opinión, de la vinculación entre el para qué, el para quién, y el cómo en la E-A de la IC.

\section{El ámbito de lo sanitario y los retos compartidos}

Las presentes reflexiones se sitúan en el campo de la salud, y más concretamente en el de los servicios sanitarios públicos. Mis limitadas experiencias en investigación y docencia en IC a lo largo de los últimos veinte años han estado siempre acompañadas de la labor asistencial como médico de familia en un Centro de Atención Primaria del Servicio Vasco de Salud, en la periferia de San
Sebastián. La investigación en Atención Primaria en nuestro contexto se ha sustentado tradicionalmente en la voluntad de los propios interesados, sin apenas soporte estructural y sin vinculación con el medio universitario ${ }^{7}$. En consecuencia, han sido numerosos los llamamientos a investigar los problemas de salud y de su atención allí donde se presentan de forma mayoritaria y donde su complejidad se hace más visible ${ }^{8}$, advirtiéndose en los últimos años, y a pesar de su todavía parcial desarrollo, un creciente reconocimiento de su importancia ${ }^{9}$. En este sentido, en España ha tenido lugar una progresiva incorporación de las metodologías cualitativas en los programas de formación promovidos desde las Escuelas e Instituciones de Enfermería, Unidades Docentes y Sociedad de Medicina Familiar y Comunitaria, y Centros y Escuelas de Salud Pública, con las consiguientes oportunidades, en mi caso, de participar y aprender de dichas iniciativas.

A su vez, a nivel internacional también se ha producido un importante desarrollo del campo concreto de la IC en Salud (ICS), con numerosos encuentros, colaboraciones en red, publicaciones y Congresos, y no solo entre países e instituciones de habla inglesa. Concretamente en nuestro medio los Congresos Iberoamericanos de ICS han supuesto una viva expresión de los avances logrados y de las posibilidades de compartir reflexiones e inquietudes. De hecho el presente artículo se basa principalmente en lo expuesto en el IV Congreso Iberoamericano de ICS que tuvo lugar, con gran éxito de participantes y de contenidos, en septiembre de 2010 en Fortaleza (Brasil).

Comenté entonces lo preocupante que en mi opinión resultaba la participación minoritaria de profesionales de la salud involucrados directamente en la asistencia sanitaria. En sondeos informales en algunas de las sesiones, la mayoría de los asistentes procedían del ámbito académico, y sobre todo de escuelas y facultades de enfermería y, en menor medida, de trabajo social, psicología y medicina.

Por supuesto, resulta indiscutible que la investigación y la mejora de la salud de los individuos y de las poblaciones no es patrimonio de ninguna profesión, disciplina o institución, y que el papel de la academia resulta clave en la formación de los futuros profesionales. También es cierto que, hoy por hoy, el grueso de las inversiones en investigación sanitaria en los servicios de salud continúan orientadas a la parcela de lo biomédico, promovidas por intereses no siempre vinculados a la satisfacción de las necesidades reales de las personas, y ajenas en gran medida a 
las realidades sociales, culturales, económicas y políticas que condicionan los niveles de salud y de bienestar.

Pero, a pesar de la influencia de sus efectos en los contenidos y metodologías de las investigaciones y publicaciones sanitarias, tampoco podemos ignorar el papel de los propios profesionales en el mantenimiento o en el cambio de dichas tendencias y la necesidad de su participación activa "desde dentro" de los servicios de sa$\operatorname{lud}^{10}$. En este sentido, resulta de especial importancia buscar vías que contribuyan a la apertura de perspectivas en el conocimiento científico-sanitario incluyendo la incorporación de las metodologías cualitativas en sus prácticas investigadoras más allá del medio universitario y del limitado periodo de formación académica.

Sin duda las dificultades son muchas y de diferente naturaleza, lo cual entiendo que justifica aún más la pertinencia del debate y del intercambio de inquietudes desde enfoques que contemplen tanto los retos compartidos en la E-A de la IC, como las particularidades de su desarrollo teórico y práctico en el ámbito de los servicios de salud.

\section{Breve descripción de una experiencia on-line en atención primaria}

A modo de referente práctico, y novedoso en algunas de sus particularidades, resumiré brevemente las características de una experiencia formativa on-line en la que hemos participado un equipo pluridisciplinario a lo largo ya de ocho años, y que nos servirá para señalar a continuación algunos posibles ejes de discusión acerca de la E-A de la IC con profesionales sanitarios ${ }^{11}$.

Existen pocas referencias publicadas sobre actividades de E-A en IC dirigidas explícitamente a profesionales de atención primaria y mantenidas a lo largo del tiempo ${ }^{12}$. En nuestro caso, el Curso de Introducción a la Investigación Cualitativa en Atención Primaria ha formado parte, desde su inicio en el año 2003, del Máster de Investigación en Atención Primaria organizado por la SemFYC, la Fundación Dr. Robert y la UAB como E-A on-line ${ }^{13}$.

Los alumnos pueden conectarse a un campus virtual propio desde cualquier lugar para acceder a los materiales, cumplimentar las evaluaciones, contactar con los profesores y participar en los Foros de discusión para el intercambio de dudas y sugerencias.

Los contenidos del Curso se han ido actualizando periódicamente y en el último año pasa- ron de 5 a 8 Unidades con el objeto de adecuarse a los requisitos de los planes de estudio europeos: 1) Aspectos teóricos y ámbitos de aplicación, 2) El diseño de la investigación cualitativa. principios generales, fases y decisiones, 3) Muestreo y selección de fuentes de información 4) Técnicas de generación de información I, 5) Técnicas de generación de información II, 6) Análisis y la interpretación de la información, 7) Los programas informáticos en la investigación cualitativa, y 8) Presentación y evaluación de la investigación cualitativa.

La opción por un equipo pluridisciplinario ha sido una característica importante de los Cursos. En la última edición hemos participado dos médicos de familia, una médica especialista en salud pública, dos enfermeras, un sociólogo y una farmacéutica, y desde el comienzo dicha pluralidad ha contribuido a enriquecer el desarrollo del proceso de E-A también para los propios docentes.

Como en el resto de los Cursos del Máster el participante dispone en cada Unidad de preguntas de autoevaluación y de evaluaciones externas. Después de cada Unidad y al finalizar el Curso se emiten las calificaciones correspondientes (Excelente, Muy Bien, Bien y Regular), y a su vez se solicita a los alumnos que lleven a cabo una valoración del Curso conforme a un formato de respuestas abiertas a un guión orientativo (contenidos de las Unidades y de las Evaluaciones, conocimientos recibidos y accesibilidad de los profesores, utilidad de lo aprendido, dinámica del Curso, adecuación del soporte informático, calendario-tiempo, y otras posibles consideraciones). El coordinador de Curso elabora a partir de dichas evaluaciones un informe que distribuye al conjunto de los profesores y envía asimismo a los directores y organizadores del Máster.

En cuanto a la profesión de los participantes, hasta la fecha la mayoría han sido médicos de familia, algunos de ellos vinculados a la docencia y otros en periodo de especialización, contando también con profesionales de enfermería, fisioterapia, epidemiología y farmacia.

En la Tabla 1 se resumen los datos referidos al número de alumnos matriculados y que finalizaron el Curso en cada año, las calificaciones emitidas y el número de evaluaciones de los Cursos elaboradas y enviadas por los alumnos (del primer año no se cuenta con información).

Como se puede observar, la demanda no ha seguido un patrón regular, habiéndose estabilizado en los últimos años en torno al $70 \%$ el porcentaje de alumnos matriculados que finali- 
Tabla 1. Curso de Introducción a la Investigación Cualitativa en Atención Primaria. 2003-2011.

\begin{tabular}{lccccccc}
\hline & & & \multicolumn{4}{c}{ Calificaciones } & Evaluaciones \\
\cline { 4 - 6 } Curso - año & Matriculados & Finalizados & E & MB & B & R & n \\
\hline $2003-2004$ & 29 & 8 & 1 & 3 & 3 & & 18 \\
$2004-2005$ & 61 & 27 & 2 & 13 & 12 & & 11 \\
$2005-2006$ & 60 & 23 & 2 & 10 & 9 & 2 & 23 \\
$2006-2007$ & 57 & 36 & 1 & 14 & 21 & & 14 \\
$2007-2008$ & 35 & 20 & 2 & 8 & 10 & 11 & 25 \\
$2008-2009$ & 23 & 16 & & 5 & 11 & 8 \\
$2009-2010$ & 37 & 29 & 1 & 8 & 18 & 2 & 113 \\
$2010-2011$ & 17 & 12 & 1 & 3 & 8 & & \\
total & 319 & 171 & & & & & \\
\hline
\end{tabular}

zan los cursos. La dificultad de compatibilizar el estudio con el trabajo asistencial, con la vida familiar, y con la participación en muchos de los casos en otros Cursos del Máster, ha sido la razón más frecuentemente señalada por los propios alumnos. El porcentaje de evaluaciones de los cursos elaboradas y remitidas por los alumnos ha oscilado entre el 48 y el $85 \%$, generando un total de 113 documentos conocidos y comentados a su vez por los docentes a quienes también se les consultó acerca de sus vivencias en relación a los Cursos. A partir de dichos textos se han identificado cuatro ejes temáticos principales que se exponen a continuación a modo de guía para el debate. No se trata por tanto de evaluar dicha experiencia formativa, sino de tomarla como excusa, a modo de ejemplo cercano, para reflexionar y discutir sobre las peculiaridades de la E-A de la IC en el medio sanitario.

\section{Algunos ejes de discusión}

Como veremos seguidamente, en la revisión temática de los textos surgen un conjunto de vivencias, exigencias y dificultades, en parte compartidas con las expuestas por otros autores en el ámbito académico, pero a su vez indicativas de un contexto y unos protagonistas diferenciados.

\section{1) Plataformas informáticas y soportes formativos}

De entre ellas, una primera es la relativa al soporte on-line de la experiencia y al papel que en este sentido las tecnologías de la información y comunicación (TICs) pueden desempeñar en la E-A de la IC. En nuestro caso, las valoraciones han sido en general positivas en todas las ediciones.

El soporte informático me ha sorprendido por lo bien estructurado que está, por la facilidad de acceso y el hecho de no haber fallado. H8C7.
Siempre y cuando el funcionamiento y mantenimiento de la plataforma informática estén garantizados -lo cual conlleva profesionalidad y recursos específicos-, la E-A on-line representa una gran oportunidad cara a facilitar la disponibilidad de materiales docentes y la adecuación del proceso e aprendizaje a las circunstancias de vida y trabajo de los alumnos.

El factor de que sea un curso on-line me ha hecho posible que lo realizara; no puedo ir a cursos presenciales por mis cambios continuos de horarios en el trabajo. M2C6.

Sin embargo, resulta innegable la importancia de la interacción entre alumnos y profesores en la E-A de la IC, por lo que en ausencia de sesiones presenciales que encarecerían y dificultarían su factibilidad al menos en nuestro medio, se requiere de un plus de disponibilidad y accesibilidad por parte de los docentes.

Cuando se plantearon dudas en el foro o a través del buzón del profesor las respuestas nunca se hacian esperar. Es una de las grandes ventajas de este formato. H6C7.

La accesibilidad ha sido perfecta... Buen trabajo. Muy intenso, ágil, rápido, constantes mensajes a diario, no nos habéis dejado olvidarnos de lo que hacíamos, permanentemente tutorizados. Muy bien... H2C6

Como ocurre con otras modalidades formativas, las TICs constituyen instrumentos con un gran potencial de ayuda en la E-A de la IC. Más allá de sus aplicaciones concretas en áreas como la generación de información o el análisis de los datos cualitativos, sobre las que existe abundante literatura, experiencias como la descrita de E-A on-line pueden servir de ejemplo cara a facilitar el acceso al conocimiento de la IC por parte de profesionales sanitarios con problemas de desplazamiento o de disponibilidad de horarios fijos. Pero también aquí conviene no engañarse acerca de lo 
que las TICs pueden y no pueden proporcionar. Además de los medios necesarios para garantizar el buen funcionamiento de las plataformas informáticas, su mera existencia no debería contemplarse en ningún caso como sustitución o alternativa al compromiso e implicación de alumnos y profesores. Implicación que no resulta fácil mantener a lo largo del tiempo, y que por tanto ha de ser promovida y cuidada de modo especial.

\section{2) La vinculación a la práctica}

En lo que respecta a los contenidos y las dinámicas relacionadas con la IC como objeto de la E-A con profesionales sanitarios, quizás uno de los aspectos principales a tener en cuenta sea la importancia de su vinculación con la actividad práctica. Se trata de personas cuya labor habitual tiene lugar en contacto con los problemas de salud y de enfermedad de los individuos y las poblaciones, por lo que resulta inevitable que ante la IC como metodología de investigación se pregunten para qué sirve, en qué les puede ayudar, y cómo aplicarla en su medio.

Creo...que tengo herramientas para empezar a hacer alguna cosita. En el ambulatorio ya queremos montar algo...sobre las dificultades para el seguimiento de la dieta, el ejercicio y la medicación en diabéticos mal controlados de origen marroquí. M5C4.

Globalmente, el módulo ha ultrapasado mis expectativas. Los conocimientos adquiridos en este módulo ya están siendo útiles en mi práctica.H1C3

Afortunadamente contamos hoy por hoy con una gran producción de investigaciones cualitativas en salud que han mostrado su relevancia para el conocimiento y la mejora de problemas relacionados con la salud de los pacientes, con las prácticas profesionales, y con el funcionamiento de las organizaciones y servicios sanitarios ${ }^{14}$. El reconocimiento de la complejidad de las intervenciones sanitarias y el surgimiento de iniciativas de evaluación de su efectividad como alternativa a las limitaciones del modelo Cochrane ${ }^{15,16}$, representan importantes oportunidades para incorporar dicho acervo en el diseño y en los contenidos de la E-A orientada a los profesionales sanitarios.

Como veremos a continuación, la orientación a la práctica no debería entrar en contradicción con el papel imprescindible de la teoría y de la reflexión, en profundidad y en diversidad, sobre lo que aportan las metodologías cualitativas al conocimiento y a la investigación en salud. Ciertamente, las tendencias a considerar "lo teórico" como algo ajeno y propio de otras disciplinas presentan un notable arraigo.
Alguna unidad me ha resultado muy difícil de entender, de poder "sacar" consecuencias prácticas...El trabajo práctico me generó bastante dificultad... Creo que este módulo debería adaptarse a profesionales que no son psicólogos o sociólogos. Definitivamente creo que los conocimientos adquiridos me serán útiles. M2C2.

Pero precisamente por ello, la apuesta por la complementariedad constituye un reto fundamental en la E-A de la IC en el medio sanitario.

3) Teoría, descubrimiento/métodos y rigor

En efecto, y como consecuencia en parte del exceso de "practicismo" en las profesiones sanitarias, la incorporación de la reflexión teórica y epistemológica en la E-A de la IC no es en general tarea fácil. La "naturalización" del enfoque positivista de la ciencia y de la investigación en el campo de la salud, favorece la tendencia al protocolo deductivo, a la marginación del debate teórico, y al riesgo de reducir la metodología de la investigación al nivel de lo procedimental ${ }^{2,6}$.

Se trata por tanto, no sólo de transmitir información acerca de unas metodologías desconocidas para la mayoría, sino sobre todo de ayudar a generar actitudes de apertura en los modos de conocer e investigar, y de cuestionamiento de la linealidad causal predominante en el pensamiento biomédico.

Para mi la investigación cualitativa ha sido una novedad. Nunca habia oido hablar de ella. Reconozco que me ha costado bastante cambiar el chip y aprender a razonar desde el punto de vista cualitativo, a veces mas cercano a la sociología o filosofía que a la medicina. M3C7

En gran medida ello conlleva una cierta consciencia de nadar contra corriente ${ }^{1}$, y a su vez requiere de una adecuación tanto de objetivos y expectativas como de contenidos. En este sentido el reto es doble ya que por un lado la información ha de ser lo suficientemente asequible como para ser comprendida, pero por otro, no ha de ocultar el esfuerzo que supone aprender a investigar y las dificultades inherentes a la IC como perspectiva metodológica. Sin duda ello supone un trabajo especial en el diseño de los materiales y en la dinámica docente, y también un esfuerzo en ocasiones mayor de lo esperado por parte de los alumnos.

A mí, en concreto, me ha costado más tiempo y esfuerzo estudiar y asimilar el materia porque estaba poco familiarizado con la metodología, y las evaluaciones han sido exigentes (lo valoro como una virtud, ya que sin exigencia no hay progreso). Tal vez me ha faltado profundizar algo en los con- 
ceptos y en los trabajos, pero el tiempo disponible es finito.... En fin, que me ha gustado mucho el módulo, he aprendido, y me ha enseñado la perspectiva cualitativa de la investigación, por lo menos intuirla y empezar a explorarla. H3C8

La tensión entre la reflexión teórica y el aprendizaje de habilidades prácticas recorre todos y cada uno de los momentos o fases de la IC, y por lo tanto también de su E-A.

Desde su justificación como opción metodológica, y pasando por el diseño y vías de generación de información, así como por los diferentes modelos y estrategias de análisis, las discusiones sobre el cómo, el porqué y el para qué, deben formar parte imprescindible del proceso de E-A.

Quizás uno de los ámbitos en que dicha tensión resulta más evidente sea el de la evaluación de la calidad de las IC ya que en la mayoría los casos la vinculación futura de los alumnos con la IC va a quedar limitada a la lectura de artículos fruto de investigaciones ajenas, lo cual debe ser tenido especialmente en cuenta. Los extremos opuestos del "todo vale" y de la asunción mecánica de los check-lists procedentes de los estudios experimentales, delimitan un campo sin duda plural y abierto, pero que no puede ser obviado en un contexto como el sanitario donde preguntarse por las evidencias forma parte inexcusable de la buena práctica. El cuestionamiento crítico de las jerarquías de evidencias difundidas junto a la MBE como criterios de ciencia o de verdad ${ }^{17}$, y la importancia de no perder de vista "lo sustantivo" de la $\mathrm{IC}^{18}$ en la evaluación de su calidad, representan campos de reflexión y de debate teórico que en mi opinión no deberían faltar en la EA de la IC en el medio sanitario actual.

\section{4) Diálogos múltiples y reflexividades}

Apuntaba anteriormente la importancia de lo pluridisciplinario en nuestra experiencia docente. El ejercicio de convergencia de distintas procedencias profesionales y formativas, y también de diferentes grados y enfoques en los conocimientos y en las prácticas de la IC, ha supuesto al menos en mi caso, y creo que para todos, una importante fuente de aprendizaje.

Cada docente se responsabiliza al menos de una Unidad, y por lo tanto se requiere de una comunidad de criterios básica y de coordinación entre los docentes para que el programa del Curso resulte coherente. Pero a su vez dicha diversidad constituye una oportunidad y un reto también para los alumnos.

En cuanto a las Unidades, cada una de ellas de manera independiente me han parecido producti- vas y diferentes, mostrando características de cada uno de vosotros, pero al mismo tiempo homogéneas y muy bien coordinadas.H2C3.

La interacción con los otros y el reconocimiento de las diferencias forma parte esencial de la IC y también de su E-A. Las interacciones y los diálogos son además múltiples ya que la mayoría de los alumnos en nuestro caso participan también de los demás Módulos de estadística y epidemiología que configuran el Máster en su conjunto.

En este sentido, optar por lo pluridisciplinario no siempre es fácil, ya que en cierta medida supone caminar en sentido contrario a las tendencias predominantes de superespecialización y parcelación de saberes, y aunque los recorridos son sin duda enriquecedores, se trata de zonas de cruce, en general periféricas respecto de cada disciplina, y por lo tanto más incomodas y "menos seguras" que las situadas en sus núcleos más centrales y más mono-identitarios.

Por otro lado, dicho pluralismo -que no equivale al eclecticismo del todo vale para todo- se corresponde en gran medida con la complejidad y heterogeneidad del medio sanitario en cuanto objeto de estudio $^{19}$. Su plasmación en la E-A de la IC requiere tener en cuenta los riesgos de la excesiva simplificación, y del modo de pensar dicotómico (bueno/malo, correcto/incorrecto), pero también la importancia de los lenguajes como medio de interlocución.

Insisto en lo complicado del lenguaje, en cierta medida en todas y cada una de las Unidades, especialmente destacable en la cuarta. H3C3.

Aunque me ha resultado muy complicado este módulo por el desconocimiento total sobre el mismo y el lenguaje utilizado en ocasiones, que me obligaba a leer y releer, finalmente tengo la sensación de que he aprendido mucho... y que el esfuerzo ha merecido la pena. M1C8.

El curso creo que ayuda a tomar conciencia de eso que llamaba "narratividad regulada", es decir, la necesidad de encontrar un terreno de encuentro "entre" la concisión (aparentemente denotativa) del lenguaje sanitario...y la narratividad hiperabierta (aparentemente hiperconnotativa...) del lenguaje de las ciencias sociales... DOC3.

Las dinámicas de interacción entre diferentes desempeñan un especial protagonismo en la IC y, por lo tanto, en su E-A. En este sentido, considero necesario incluir al menos un breve apunte en relación a la reflexividad. Como señala Hammersley, por parte de algunas de las corrientes de E-A el ejercicio de la reflexividad apenas se contempla, e incluso puede llegar a valorarse como 
algo negativo ${ }^{6}$. En mi opinión, si embargo, la EA de la IC representa una gran oportunidad para promover la necesaria consciencia de uno mismo en la construcción del conocimiento ${ }^{20}$. La incorporación del "yo contextualizado" en los procesos de investigación conlleva no sólo el cuestionamiento de la pretendida neutralidad de la ciencia, sino además la necesidad de preguntarse por las implicaciones éticas de la propia práctica investigadora $^{21}$. Implicaciones para con los participantes en la investigación en sus distintos niveles y para con los usuarios potenciales de sus resultados, que en el medio sanitario resultan de especial trascendencia, y que por tanto deberían ser objeto de especial atención en la E-A de la IC cuando ésta se lleva a cabo con profesionales de dicho medio.

\section{Agradecimientos}

Deseo expresar mi agradecimiento a Fernando Conde, María José Fernández de Sanmamed, Olga Monistrol, Mariona Pons, Enriqueta Pujol y Amaia Sáenz de Ormijana, por su labor como compañeros docentes del Curso de Introducción a la Investigación Cualitativa en Atención Primaria; a los organizadores del Máster y a los alumnos que nos han enviado sus valoraciones; y a Malu Bosi y al Comité Organizador del IV Congreso Iberoamericano de ICS por darme la oportunidad de exponer y compartir las presentes reflexiones. 


\section{Referencias}

1. Eakin JM, Mykhalovskiy E. Teaching Against the Grain: A Workshop on Teaching Qualitative Research in the Health Sciences. Conference Report: A National Workshop on Teaching Qualitative Research in the Health Sciences [43 paragraphs] Forum Qualitative Sozialforschung / Forum: Qualitative Social Research. [periódico na Internet]. 2005 [acessado 2012 fev 17]; 6(2): Art. 42: [cerca de 15 p.]. Disponível em: http://nbn-resolving.de/urn:nbn:de:0114-fqs0502427.

2. Breuer F, Schreier M. Issues in Learning About and Teaching Qualitative Research Methods and Methodology in the Social Sciences. Forum QualitativeSozialforschung / Forum: Qualitative Social Research. [periódico na Internet]. 2005 [acessado 2012 fev 17]; 8(1): Art. 30: [cerca de 15 p.]. Disponível em: http:/ /nbnresolving.de/urn:nbn:de:0114-fqs0701307.

3. Mercado FJ, Bosi ML, Robles L, Weisenfeld E, Pla M. La enseñanza de la investigación cualitativa en salud. Voces desde Iberoamérica. Salud Colectiva 2005; 1(1):97-116.

4. Chenail RJ. Teaching and Learning Qualitative Research: Articles, Book Chapter, Books, and Web Sites. [Site na Internet]. 2012 [acessado $2012 \mathrm{fev}$ 17]. Disponível em: http://www.novaedu/ssss/QR/teaching $022211 \mathrm{pdf} 2011$

5. Centre for Critical Qualitative Health Research.University of Totonto. Teaching Qualitative Research Methods. Bibliography. [Site na Internet]. 2012 [acessado $2012 \mathrm{fev}$ 17]. Disponível em: http://www.ccqhr.utoronto.ca/teaching/publicationsteaching

6. Hammersley M. Teaching qualitative method: Craft, profession, or bricolage? In: Seale C, Gobo G, Silverman $\mathrm{D}$, editores. Qualitative research practice. London: Sage; 2004. p. 549-560.

7. Palomo L. La investigación y la evolución reciente de la atención primaria. Gac Sanit 2002; 16(2):182187.

8. Diogène-Fadini E, Plataforma para la Promoción de la Investigación APS. Promoción de la investigación clínica en atención primaria. Aten Primaria 2005; 36(10):563-565.

9. Sanchez-Celaya del PM. Investigación en Atención Primaria: continuar avanzando. Aten Primaria 2008; 40(1):3-4.

10. Morse JM. How different is qualitative health research from qualitative research? Do we have a subdiscipline? Qual Health Res 2010; 20(11):1459-1464.

11. Calderón C, Conde F, Fernández de Sanmamed MJ, Monistrol O, Pons M, Pujol E, Sáenz de Ormijana A. Curso de Introducción a la Investigación $\mathrm{Cu}$ alitativa. Máster de Investigación en Atención Primaria. Barcelona: Universitat Autònoma de Barcelona, Fundació Doctor Robert; 2010.
12. Featherstone VA, Barbour RS, Garner J. A reflection on ten years experience of providing qualitative research training in primary care. Primary Health Care Research and Development 2007; 8(3):198-206.

13. Jiménez J, Argimón JM, Calderón C, Grau O, Ferrando J. Máster de Investigación en Atención Primaria. [Documento na Internet]. 2012 [acessado 2012 fev 17]; [cerca de 8 p.]. Disponível em: http://www. semfyc.es/es/agenda/eventos/M\%E1ster+y+cursos +de+investigaci $\% \mathrm{~F} 3 \mathrm{n}+\mathrm{en}+$ Atenci $\% \mathrm{~F} 3 \mathrm{n}+$ Primaria/

14. Morse JM. What is the domain of qualitative health research? Qual Health Res 2007; 17(6):715-717.

15. Mays N, Pope C, Popay J. Systematically reviewing qualitative and quantitative evidence to inform management and policy-making in the health field. J Health Serv Res Policy 2005; 10(Supl.1):6-20.

16. Pawson R, Greenhalgh T, Harvey G, Walshe K. Realist review - a new method of systematic review designed for complex policy interventions. $J$ Health Serv Res Policy 2005; 10(Supl.1):21-34.

17. Calderón C. Evaluación de la calidad de la investigación cualitativa en salud: criterios, proceso y escritura. Forum Qualitative Sozialforschung / Forum: Qualitative Social Research [periódico na Internet]. 2009 [acessado $2012 \mathrm{fev}$ 17]; 10(2): Art. 17: [cerca de 15 p.]. Disponível em: http://nbn-resolving.de/ urn:nbn:de:0114-fqs0902178.

18. Eakin JM, Mykhalovskiy E. Reframing the evaluation of qualitative research: reflections on a review of appraisal guidelines in the health sciences. $J$ Eval Clin Pract 2003; 9(2):187-194.

19. Beltrán M. Cuestiones previas acerca de la ciencia de la realidad social. En: García F, Ibáñez J, Alvira F, editores. El análisis de la realidad social. Métodos y técnicas de investigación.Madrid: Alianza Editorial SA; 1986. p. 17-29.

20. Finlay L. Outing the researcher: The provenance, process and practice of reflexivity. Qual Health Res 2002; 12(4):531-545.

21. Calderón C. El papel de la reflexividad en la investigación cualitativa en salud. Rev Clínica Electrónica en Atención Primaria. No prelo 2011.

Apresentado em 06/09/2011

Aprovado em 27/12/2011

Versão final apresentada em 10/01/2012 